\title{
COLONOSCOPIA COMO MÉTODO DIAGNÓSTICO E TERAPÊUTICO DAS MOLÉSTIAS DO INTESTINO GROSSO: análise de 2.567 exames
}

\author{
Sergio Carlos NAHAS, Carlos Frederico Sparapan MARQUES, Sérgio Alonso ARAÚJO, \\ Adilson Akihide AISAKA, Caio Sérgio Rizkallah NAHAS, \\ Rodrigo Ambar PINTO e Desidério Roberto KISS
}

RESUMO - Racional - Com o surgimento das fibras ópticas na área médica, houve grande avanço tecnológico na observação do aparelho digestivo, com a introdução dos endoscópios flexíveis, usados anteriormente para o trato digestivo superior. Objetivo - Rever os resultados da aplicação da colonoscopia diagnóstica e terapêutica na Disciplina de Coloproctologia da Faculdade de Medicina da Universidade de São Paulo, respeitando as características de instituição de ensino e aprimoramento médico. Casuística e Métodos - Análise retrospectiva dos dados referentes a 2.567 exames de fibrocolonoscopia realizados entre os anos de 1984 e 2002, seja em regime de internação hospitalar ou ambulatorial. As principais indicações de exame nesta casuística foram o sangramento retal/anemia em 571 (22,24\%) doentes, alteração do hábito intestinal em 379 (14,76\%), moléstia inflamatória em 222 (8,65\%) e pós-operatório de neoplasia em 186 (7,25\%). O preparo intestinal com manitol foi realizado na maioria dos doentes. A sedação, quando não contra-indicada, foi feita com meperidina e benzodiazepínico. Todos exames foram feitos com monitorização com oxímetro de pulso. Resultados - O resultado do exame foi normal em 1.089 (42,42\%) casos. Pólipos foram diagnosticados em 397 (15,47\%) casos, doença diverticular em 330 (12,86\%), doença inflamatória em 305 (11,88\%) e câncer colorretal em 262 (10,21\%). Foram realizadas 819 polipectomias em 397 doentes, gerando a média de 2,21 polipectomias por doente com pólipo. A colonoscopia foi considerada incompleta (quando não atingiu o ceco) em 181 $(7,05 \%)$ casos. Houve um caso de perfuração por fratura de tumor subestenosante de retossigmóide. Complicações relacionadas à sedação levaram à interrupção do exame em $0,42 \%$ das vezes, sem maiores prejuízos aos pacientes. Conclusão - A colonoscopia foi método eficaz no diagnóstico e tratamento de afecções colorretais, sendo seguro e com baixo índice de morbidade em ambiente universitário.

DESCRITORES - Enteropatias, diagnóstico. Colonoscopia. Pólipos intestinais, diagnostico.

\section{INTRODUÇÃO}

Desde 1960, com o surgimento das fibras ópticas na área médica, houve grande avanço tecnológico na observação do aparelho digestivo, com a introdução dos endoscópios flexíveis, usados anteriormente para o trato digestivo superior ${ }^{(20)}$.

A visualização completa da mucosa do intestino grosso através da colonoscopia é sabidamente de fundamental importância para o diagnóstico correto da maioria das afecções colorretais ${ }^{(1)}$, com poucas exceções, como megacólon e doença diverticular na crise aguda.

Atualmente destaca-se como um dos métodos mais completos de investigação das doenças colorretais ${ }^{(17,19}$, ${ }^{20)}$, com vantagens sobre outros métodos de investigação por proporcionar a observação da mucosa colônica e muitas vezes do íleo terminal, em tempo único e de forma direta ${ }^{(19)}$.

$\mathrm{O}$ incessante aperfeiçoamento de tecnologias de videoendoscopia, de informática e o desenvolvimento de recursos auxiliares como a cromoscopia e magnificação de imagem são de contribuição inexorável para a aplicação mais ampla do método, beneficiando maior número de pacientes. Tais tecnologias, ainda que custosas, tornam a execução do procedimento menos difícil. Outra vantagem da aplicação destas tecnologias é a maior segurança associada à realização do exame colonoscópico seja para diagnóstico, ou tratamento ${ }^{(18)}$.

O presente estudo teve por objetivo rever os resultados da aplicação da colonoscopia diagnóstica e terapêutica na Disciplina de Coloproctologia da Faculdade de Medicina da Universidade de São Paulo, respeitando as características de instituição de ensino e aprimoramento médico. 


\section{CASUÍSTICA E MÉTODOS}

Foram submetidos a análise retrospectiva os dados referentes a 2.567 exames de colonoscopia realizados entre os anos de 1984 e 2002, seja em regime de internação hospitalar ou de forma ambulatorial. Foram computados dados relativos a indicações da realização dos exames, diagnóstico endoscópico, morbidade e mortalidade associadas ao método empregado, freqüência e motivos de exames incompletos e resultados da realização de procedimentos terapêuticos - polipectomias.

Para o preparo intestinal foi utilizada solução de manitol ${ }^{\circledR}$ a $10 \%{ }^{(13)}$. Na véspera do exame, o paciente era instruído a ingerir livremente dieta sem resíduos e quatro comprimidos $(20 \mathrm{mg})$ de bisacodil ${ }^{\circledR}$ ao final do dia. Na manhã do exame, recebia $10 \mathrm{mg}$ de metoclopramida via intramuscular seguida de ingestão de $500 \mathrm{~mL}$ de solução de manitol a $10 \%$. Pacientes com suspeita ou quadro clínico de suboclusão intestinal foram excluídos do método.

Antibioticoprofilaxia foi realizada somente para pacientes com alto risco para endocardite infecciosa ou portadores de prótese valvar cardíaca, conforme recomendado pela Sociedade Americana de Endoscopia Gastrointestinal ${ }^{(28)}$ e pela Associação Americana de Cardiologia ${ }^{(9)}$. Esses doentes receberam a associação de ampicilina e gentamicina.

Pacientes em uso de terapia anticoagulante tiveram suspensão temporária da medicação. Doentes em uso de antiagregantes plaquetários ou antiinflamatórios não-hormonais tiveram seu uso suspenso por 7 dias previamente a data da realização do exame.

A sedação para a realização da colonoscopia foi realizada de forma seletiva e para a maioria dos pacientes. Para os doentes já submetidos a sigmoidectomias prévias, o exame sem sedação constituiu a opção inicial. Nos demais casos, foi realizada associação do analgésico meperidina (dose inicial de $50 \mathrm{mg}$ ) com o sedativo diazepam $(5-10 \mathrm{mg}$ ) ou midazolam (4-15 mg) administrados por via endovenosa. A administração do antagonista opióide naloxone (2-4 mg) foi feita conforme opção do endoscopista ao término do procedimento e baseada na dose de meperidina administrada, bem como o nível de consciência do paciente na ocasião.

O antifisético dimeticona foi eventualmente administrado, diluído à solução de irrigação através do canal do colonoscópio para eliminar bolhas decorrentes do uso do manitol $l^{\circledR}$.

Os endoscópios utilizados foram dois fibrocolonoscópios da marca Olympus ${ }^{\circledR}$ e Fujinon ${ }^{\circledR}$, sendo que um deles possuía o recurso de magnificação de imagem. Empregou-se a fonte de luz de $200 \mathrm{~W}$. Quando necessária, utilizou-se eletrocoagulação por diatermia (monopolar) com a unidade eletrocirúrgica Valley Lab ${ }^{\circledR}$. As biopsias foram colhidas por pinças de biopsia a frio, tipo espiculada, e os procedimentos de polipectomia foram realizados por alça tipo ovalada, ambas da marca Olympus ${ }^{\circledR}$. Para as polipectomia de lesões pequenas (até $5 \mathrm{~mm}$ ), a pinça de biopsia a frio foi usada com a vantagem da coleta do material para exame histológico. A excisão a frio foi realizada para esse tipo de lesão como opção do endoscopista frente aos relatos de complicações decorrentes da "hot biopsy". A alça de polipectomia tipo ovalada foi empregada para lesões pediculadas maiores que $5 \mathrm{~mm}$ ou em pólipos sésseis para as ressecções do tipo fatiada ("piecemeal") ou após realizar coxim submucoso com soro fisiológico.

\section{RESULTADOS}

A principal indicação de exame colonoscópico nesta casuística foi o sangramento retal/anemia, ocorrendo em 571 (22,24\%) doentes, seguida pela alteração do hábito intestinal em 379 $(14,76 \%)$ casos (Tabela 1$)$.

TABELA 1 - Indicações de colonoscopia em 2.567 doentes

\begin{tabular}{lcc}
\hline Indicação & $\mathbf{n}$ & $\%$ \\
\hline Sangramento retal/anemia & 571 & 22,24 \\
Alteração do hábito intestinal & 379 & 14,76 \\
Doença inflamatória & 222 & 8,65 \\
Seguimento de câncer operado & 186 & 7,25 \\
Antecedente de neoplasia & 172 & 6,70 \\
Colite & 134 & 5,22 \\
Emagrecimento & 130 & 5,06 \\
Diarréia & 121 & 4,71 \\
História familiar de câncer colorretal & 111 & 4,32 \\
Enema opaco inconclusivo & 98 & 3,82 \\
Antecedente de pólipo & 75 & 2,92 \\
Seguimento de polipectomia & 66 & 2,57 \\
Rastreamento de neoplasia & 57 & 2,22 \\
Dor abdominal & 53 & 2,06 \\
Pólipo em exame & 51 & 1,99 \\
Polipose colônica & 45 & 1,75 \\
Pesquisa de tumor sincrônico & 21 & 0,82 \\
Proctite & 10 & 0,39 \\
Seguimento de polipose colônica operada & 8 & 0,31 \\
Fístula retovaginal & 5 & 0,19 \\
Massa abdominal & 4 & 0,16 \\
Obstrução intestinal & 2 & 0,08 \\
Outros & 46 & 1,79 \\
\hline TOTAL & 2567 & 100,00 \\
\hline
\end{tabular}

Para a maioria dos doentes, o exame resultou normal em 1.089 $(42,42 \%)$ casos. O diagnóstico mais freqüentemente encontrado foi o de pólipo, em 397 (15,47\%) casos, seguidos pela doença diverticular em $330(12,86 \%)$ casos. Os diagnósticos endoscópicos encontrados estão relacionados na Tabela 2.

A sedação para realização do exame gerou complicações em seis casos que tiveram mudança do padrão respiratório e cinco com alteração do nível de consciência. Essas complicações levaram à interrupção do exame e conseqüente prejuízo ao procedimento em $0,42 \%$ das vezes, porém sem morbidades ao paciente.

Foram realizadas 819 polipectomias em 397 doentes, gerando a média de 2,21 polipectomias por doente com este diagnóstico. Não houve complicações associadas às polipectomias endoscópicas.

O exame foi considerado incompleto quando não alcançava o ceco, o que ocorreu em 181 doentes, ou seja, 7,05\% da casuística. O preparo de cólon para o exame foi considerado inadequado em $45(1,75 \%)$ das colonoscopias realizadas. As causas de exames incompletos estão listadas na Tabela 3. 
TABELA 2 - Resultados do exame endoscópico em 2.567 doentes

\begin{tabular}{lcc}
\hline Diagnóstico & $\mathrm{n}$ & $\%$ \\
\hline Normal & 1089 & 42,42 \\
Pólipos & 397 & 15,47 \\
Doença diverticular & 330 & 12,86 \\
Doença inflamatória & 305 & 11,88 \\
Câncer & 262 & 10,21 \\
Colite/retite & 72 & 2,80 \\
Polipose adenomatosa & 24 & 0,93 \\
Estenose & 21 & 0,82 \\
Megacólon & 10 & 0,39 \\
Polipose juvenil & 9 & 0,35 \\
Angiodisplasia & 9 & 0,35 \\
Preparo inadequado & 7 & 0,27 \\
Melanose & 6 & 0,23 \\
Lipoma & 3 & 0,12 \\
Compressão extrínseca & 3 & 0,12 \\
Hemangioma & 1 & 0,04 \\
Outros & 19 & 0,74 \\
\hline TOTAL & 2567 & 100,00 \\
\hline
\end{tabular}

TABELA 3 - Causas de exame colonoscópico incompleto em 2.567 doentes

\begin{tabular}{lcc}
\hline Causas & $\mathbf{n}$ & $(\%)$ \\
\hline Lesão intransponível & 82 & 45,30 \\
Preparo inadequado & 45 & 24,86 \\
Dor & 34 & 18,80 \\
Dificuldade técnica & 9 & 4,97 \\
Alteração do padrão respiratório & 6 & 3,31 \\
Alteração do nível de consciência & 5 & 2,76 \\
\hline TOTAL & 181 & 100,00 \\
\hline
\end{tabular}

Houve um caso de perfuração por fratura de tumor subestenosante da transição retossigmoidiana diagnosticada precocemente, necessitando de laparotomia de urgência para realização de retossigmoidectomia a Hartmann, com boa evolução pós-operatória e reconstrução do trânsito após 3 meses. Não houve nenhum óbito relacionado ao procedimento.

\section{DISCUSSÃO E CONCLUSÕES}

A primeira colonoscopia total realizada com sucesso utilizando fibra óptica foi reportada em 1966 por OVERHOLT e POLLARD ${ }^{(22)}$. A partir de então, com o aperfeiçoamento do método e melhor desenvolvimento técnico, esse exame despontou como o principal meio de avaliação do cólon, sendo mais sensível do que o exame radiológico e oferecendo uma gama de opções terapêuticas ${ }^{(4)}$.

Há 10 anos, o surgimento e aperfeiçoamento da tecnologia em videoendoscopia transformaram a colonoscopia em um procedimento de mais simples execução, facilitando assim, seu aprendizado. No entanto, devido ao fato de ser um exame invasivo, pode trazer certos riscos ao paciente, mesmo quando realizado por médico experiente ${ }^{(18)}$.

$\mathrm{O}$ atual estudo visou analisar retrospectivamente os resultados do emprego da fibrocolonoscopia quanto a sua eficácia e segurança, em um hospital de ensino e aprimoramento médico.
Dentre as indicações para a realização da colonoscopia, o sangramento retal e anemia constituíram as causas mais comuns para a realização do exame, sendo responsáveis por $22,24 \%(n=571)$ dos mesmos, resultando indicação freqüente e importante conforme a casuística de muitos outros autores ${ }^{(4,29)}$. A alteração do hábito intestinal foi responsável por $14,76 \%(\mathrm{n}=379)$ dos procedimentos. Essa indicação pode, em parte, explicar a alta prevalência de exames normais $(n=1089)$ na presente casuística. Outras indicações, como história familiar de câncer colorretal $(n=111)$, emagrecimento $(n=130)$, enema opaco inconclusivo $(\mathrm{n}=98)$ e dor abdominal $(\mathrm{n}=53)$ também vieram a corroborar com o alto índice de exames sem alterações. Esses achados suportam a hipótese de que a disponibilidade do método definitivo ou padrão-ouro, para a investigação do intestino grosso em um hospital-escola, pode gerar anamneses incompletas e indicações excessivas destes exames.

O diagnóstico mais freqüente nos exames realizados foram pólipos, encontrados em 397 (15,47\%) ocasiões, e para os quais foram feitas 819 polipectomias endoscópicas. Resultado semelhante foi obtido em estudo prospectivo que analisou 9.223 colonoscopias no Reino Unido, em que os pólipos foram os achados mais comuns, encontrados em 22,5\% dos casos, e a polipectomia realizada na maioria ${ }^{(4)}$. É de grande importância a detecção e tratamento adequados de pólipos, considerando que a maior parte dos carcinomas colorretais decorram de pólipos adenomatosos pré-existentes (seqüência adenoma-adenocarcinoma).

Apesar de sua alta sensibilidade, a colonoscopia pode apresentar resultados falso-negativos, fato que pode ser atribuído a impossibilidade anatômica de visibilizar o ceco, preparo intestinal inadequado ou técnica e treinamento inapropriados ${ }^{(12)}$.

A ocorrência e a gravidade das complicações também podem ser modificadas pela qualidade do preparo. Para a presente casuística houve preparo inadequado em 45 (1,75\%) doentes, dificultando a realização dos exames neles. $\mathrm{O}$ alto índice de sucesso dos preparos indicam que o emprego do manitol ${ }^{\circledR}$, além de amplamente disponível no mercado, persiste nos moldes descritos como método eficaz e seguro no preparo intestinal de doentes a serem submetidos a colonoscopia. Algumas condições freqüentemente encontradas neste estudo, como lesões intransponíveis podem ter sido responsáveis por prejuízo no preparo intestinal.

É de fundamental importância o conhecimento das complicações associadas ao uso da colonoscopia, por ser exame rotineiramente utilizado. As principais citadas na literatura são: sangramento, perfuração e alterações cardiopulmonares associadas à sedação ${ }^{(5,6,15)}$.

Sangramento e perfuração após polipectomias endoscópicas são as complicações mais freqüentes, ocorrendo respectivamente em torno de $2 \%$ e até $0,5 \%{ }^{(31)}$. Assim, as polipectomias devem ser realizadas de forma criteriosa e com técnica adequada. No presente estudo, não houve complicações decorrentes das polipectomias. Entretanto, ocorreu perfuração em um caso $(0,003 \%)$, decorrente de fratura de um tumor da transição retossigmoidiana, durante tentativa de passagem do aparelho. As perfurações colônicas 
associadas à colonoscopia são complicações mórbidas que geralmente necessitam de laparotomia de urgência, assim como neste estudo. O risco de perfuração relatado na literatura varia de $0,06 \%$ a $2,1 \%{ }^{(11)}$, considerando-se que a maioria dos casos não são relatados. FARLEY et al. ${ }^{(11)}$, em estudo retrospectivo, encontraram 43 casos de perfuração em 57.028 colonoscopias $(0.075 \%)$ ou 1 perfuração para cada 1.333 procedimentos). A maioria delas foi tratada cirurgicamente, sendo que a conduta intra-operatória foi baseada no grau de contaminação da cavidade e das condições dos tecidos.

A complicação mais freqüentemente associada à sedação é à hipóxia, que pode ocorrer entre $17 \%$ e $70 \%$ dos doentes, resultando tanto da quantidade de medicação administrada, quanto de fatores individuais (idade, insuficiência hepática ou pulmonar, anemia e instabilidade hemodinâmica). A criteriosa seleção de doentes, o uso da monitorização com oximetria de pulso, o emprego judicioso da medicação sedativa, bem como a disposição de suporte ventilatório de emergência constituem medidas eficazes na prevenção e tratamento da hipóxia ${ }^{(5,18)}$.

Por esses motivos, CATALDO et al. ${ }^{(5)}$, realizaram estudo prospectivo em que analisaram 258 pacientes submetidos a colonoscopia sem sedação, concluindo que esse procedimento é seguro, efetivo e bem tolerado pelos pacientes. Assim, essa técnica se torna interessante à medida que pode reduzir a necessidade de monitorização cardiopulmonar, não necessita de acesso venoso, pode reduzir significativamente os custos e o tempo de recuperação e pode permitir a realização do exame em pacientes que não poderiam ser submetidos a técnica sem sedação devido à presença de co-morbidades ${ }^{(5)}$.

Na presente série, foi utilizada a associação de analgésico meperidina com sedativo benzodiazepínico em baixas doses para a maioria dos doentes, o que ocasionou alteração do nível de consciência e do padrão respiratório em 11 casos $(0,42 \%)$, com conseqüente interrupção do exame. No entanto, em nenhum dos casos houve morbidade adicional ao paciente. A opção pela utilização de analgesia é explicada pela presença de aprendizes realizando os exames, ainda que supervisionados, em instituição de ensino.

A colonoscopia é um procedimento operador-dependente. De acordo com a revisão colonoscópica nacional no Reino Unido ${ }^{(3)}$, a intubação do ceco foi relata em $77,1 \%$ dos casos. Guias atuais e a literatura mundial sugerem que colonoscopistas treinados devem ter taxa de intubação do ceco de, pelo menos, $90 \%{ }^{(26)}$. No presente estudo, a intubação cecal se deu em 93,95\% dos casos. Resultados semelhantes foram obtidos em estudos feitos por THOMAS-GIBSON et al. ${ }^{(29)}$ e SIEG et al. ${ }^{(27)}$ que obtiveram taxas de intubação cecal de $93 \%$ e $97 \%$, respectivamente. As colonoscopias eram feitas por cirurgiões, clínicos, treinandos experimentados e endoscopistas especializados com taxas de $98 \%, 92 \%, 94 \%$ e $98 \%$, respectivamente, demonstrando variação de padrão segundo o grau de experiência do endoscopista ${ }^{(29)}$. Neste estudo, os exames foram praticados ou supervisionados por cirurgiões especializados em colonoscopia do Serviço de Coloproctologia do Hospital das Clínicas da Faculdade de Medicina da Universidade de São Paulo (HC-FMUSP), onde os treinandos eram residentes de $4^{\circ}$ ano de cirurgia do aparelho digestivo.

Algumas condições, como lesões obstrutivas, constrições, aderências, irradiação pélvica e doença diverticular estão associadas a dificuldades técnicas e exames menos sensíveis ${ }^{(7,8,12)}$. Nestas situações, o intestino grosso pode ser avaliado através do enema opaco, mesmo que este não seja elucidativo para certa parcela dos doentes, é alternativa diagnóstica freqüente ${ }^{(21)}$. Nova tentativa colonoscópica para estes casos deve ser baseada nas condições clínicas e no motivo pelo qual o exame não pôde ser completado. Tal conduta é embasada na constatação de que parte dos doentes em que não foi possível terminar o exame já foi submetida a colonoscopia completa pregressa ${ }^{(30)}$. Assim, para todos os doentes que tiveram preparo intestinal inadequado, a realização de novo exame torna-se uma alternativa interessante. A utilização de colonoscópios pediátricos $^{(2,14)}$ ou até de radioscopia ${ }^{(14,16)}$, sugeridos por alguns autores, pode aumentar a chance de transposição do ceco em pacientes selecionados.

As Tabelas 4 e 5 fazem análise comparativa entre alguns serviços de colonoscopia, inclusive o do HC-FMUSP, para

TABELA 4 - Análise comparativa entre serviços de colonoscopia atuais

\begin{tabular}{|c|c|c|c|c|}
\hline Serviço & $\begin{array}{c}\text { Intubação } \\
\text { cecal } \\
(\%)\end{array}$ & $\begin{array}{c}\text { Colonoscopia } \\
\text { normal } \\
(\%)\end{array}$ & $\begin{array}{c}\text { Diagnósticos } \\
\text { principais } \\
(\%)\end{array}$ & $\begin{array}{c}\text { Principais } \\
\text { indicações } \\
(\%)\end{array}$ \\
\hline Thomas-Gibson $^{(29) 1}$ & 93 & 41 & $\begin{array}{c}\text { Pólipos - 25,9 } \\
\text { DII }-17 \\
\text { DD }-9\end{array}$ & $\begin{array}{c}\text { Sgto. }-18 \\
\text { AHI - } 11 \\
\text { DII - } 11\end{array}$ \\
\hline Bowels $^{(4)}$ & 76,9 & 42,1 & $\begin{array}{c}\text { Pólipos - 22,5 } \\
\text { DD - 14,9 } \\
\text { DII - 13,9 }\end{array}$ & $\begin{array}{c}\text { Sgto. }-19,9 \\
\text { SP }-16,5 \\
\text { DII }-14,6\end{array}$ \\
\hline HC-FMUSP & 93,95 & 42,42 & $\begin{array}{c}\text { Pólipos - 15,47 } \\
\text { DD - 12,86 } \\
\text { DII - 11,88 }\end{array}$ & $\begin{array}{c}\text { Sgto. - 22,24 } \\
\text { AHI - 14,76 } \\
\text { DII }-8,65\end{array}$ \\
\hline
\end{tabular}

DII= doença inflamatória intestinal; $D D=$ doença diverticular; Sgto= sangramento;

$\mathrm{AHI}=$ alteração do hábito intestinal; $\mathrm{SP}=$ seguimento de pólipos

TABELA 5 - Complicações relacionadas a exames colonoscópicos

\begin{tabular}{|c|c|c|c|c|c|}
\hline Serviço & $\mathrm{N}^{\circ}$ exames & $\mathrm{N}^{\circ}$ polipectomias & Perfurações & Sangramento & Óbitos \\
\hline Ettersperger et al. ${ }^{(10)}$ & 5.822 & $872 * *$ & $0,20 \%-0,57 \% *$ & - & $0,03 \%$ \\
\hline Puchner et al. ${ }^{(25)}$ & 10.000 & - & $0,09 \%$ & $0,05 \%$ & 0,02 \\
\hline Parra-Blanco et al. ${ }^{(23)}$ & 9.555 & 4.735 & 0 & $1,1 \% *$ & 0 \\
\hline Sieg et al. ${ }^{(27)}$ & 82.416 & 14.249 & $0,06 \% *$ & $0,26 \% *$ & $0,07 \% *$ \\
\hline Pérez Roldan et al. ${ }^{(24)}$ & $\ldots$ & 147 & $1,36 \% *$ & $5,44 \% *$ & 0 \\
\hline Thomas-Gibson et al. ${ }^{(29)}$ & 505 & - & 0 & $0,59 \%$ & 0 \\
\hline Bowels et al. ${ }^{(4)}$ & 9.223 & & $0,065 \%$ & $0,13 \%$ & $0,065 \%$ \\
\hline HC-FMUSP & 2.567 & 819 & 0 & $0,039 \%$ & 0 \\
\hline
\end{tabular}

*relacionadas à polipectomia

**no mínimo uma polipectomia por exame 
situar-se em relação à literatura mundial. A Tabela 4 analisa as percentagens de intubação cecal, colonoscopia normal, principais indicações e diagnósticos, enquanto a Tabela 5 compara as complicações relacionadas ao procedimento do ponto de vista diagnóstico e terapêutico.

Tendo em vista os mais de 20 anos de experiência do Serviço de Colonoscopia da Disciplina de Coloproctologia da Faculdade de Medicina da USP, é possível concluir que:

- A fibrocolonoscopia nesse Serviço mostrou ser procedimento seguro, eficaz e com baixa morbidade e mortalidade nula, no diagnóstico e tratamento de afecções do intestino grosso.

- As complicações da colonoscopia tiveram natureza transitória e ocorreram, para a presente casuística, relacionadas à sedação e preparo necessários para a realização do exame.

- Os pólipos foram o diagnóstico mais freqüente, da mesma forma que na literatura mundial, o que reforça a alta prevalência dessa patologia e destaca a possibilidade terapêutica definitiva na maioria dos casos.

- A polipectomia endoscópica foi executada com sucesso e sem complicações, sendo consagrada como método terapêutico neste Serviço.

- A colonoscopia pode ser realizada por residentes em hospitalescola com supervisão direta de médicos treinados, com baixos índices de morbidade.

Nahas SC, Marques CFS, Araújo SA, Aisaka AA, Nahas CSR, Pinto RA, Kiss DR. Colonoscopy as a diagnostic and therapeutic method of the large bowel diseases: analysis of 2,567 exams. Arq Gastroenterol 2005;42(2):77-82.

ABSTRACT - Background - Since the sixties, when the optic fibers were reported, colonoscopy had emerged as the first line imaging investigation of the colon. Aim - To review the results of diagnostic and therapeutic colonoscopy at the Discipline of Coloproctology of the University of São Paulo Medical School, São Paulo, SP, Brazil, respecting the characteristics of an institution of medical eduction. Methods - Retrospective analysis of basis related to 2,567 fibro colonoscopies between 1984 and 2002. The procedure was performed in hospitalized and in outpatients. The most common indications for colonoscopy were investigation of rectal bleeding and anemia (22.4\%), change of bowel habit (14.76\%), inflammatory bowel disease (8.65\%) and carcinoma (7.25\%). Bowel preparation with manitol was used by most of the patients. Sedation, when not contra-indicated, was administered. The most common combination was meperidine and benzodiazepine. All the exams were monitored with pulse oximeter. A normal colonoscopy to the point of maximum insertion was reported in $42.42 \%$ of procedures. The most common diagnosis was polyps $(15.47 \%)$, followed by diverticular disease $(12.86 \%)$. Inflammatory disease was recorded in $11.88 \%$ and carcinoma in $10.21 \%$. Polypectomy was undertaken in 397 patients (2.21 polypectomy per patient with polyps). Colonoscopy was considered incomplete (when the colonoscope did not pass to the cecum or terminal ileum) in 181 (7.05\%) cases. Perforation was reported in one patient who had a subestenosing retossigmoid tumor. In $0.42 \%$, reasons for failing to complete the procedure included complication related to sedation, with no further prejudice for the patients. Conclusions - Colonoscopic examination of the entire colon remains the standard for visualization, biopsy and treatment of colonic affections. The incidence of complication of endoscopy of the large bowel is quite low, even in a school hospital.

HEADINGS - Intestinal diseases, diagnosis. Colonoscopy. Intestinal polyps. Polyps, diagnosis.

\section{REFERÊNCIAS BIBLIOGRÁFICAS}

1. Alves PRA, Habr-Gama A. Colonoscopia: indicações e técnica. In: Pinotti HW, ed. Tratado de clínica cirúrgica do aparelho digestivo. São Paulo: Atheneu; 1994. p.1086-90.

2. Batt L, Williams CB. Usefulness of pediatric colonoscope in adult colonoscopy. Gastrointest Endosc 1989;35:329-32.

3. Bowels CJ, Leicester R, Swabrick E, Williams CB, Romaya C, Epstein O. IntercollegiateB.S.G National Colonoscopy (IBNC) Audit: methods used to identify te caecum and caecal intubation rate [abstract]. Gut 2001;48(Supl):A10.

4. Bowles CJ, Leicester R, Romaya C, Swarbrick E, Williams CB, Epstein O. A prospective study of colonoscopy practice in the UK today: are we adequately prepared for national colorectal cancer screening tomorrow? Gut 2004;53:277-83.

5. Cataldo PA. Colonoscopy without sedation: a viable alternative. Dis Colon Rectum 1996;39:257-61.

6. Chokhavatia S, Nguyen L, Williams R, Kao J, Heavner JE. Sedation and analgesia for gastrointestinal endoscopy. Am J Gastroenterol 1993;88:393-6.

7. Cirocco WC, Rusin LC. Factors that pedrict incomplete colonoscpy. Dis Colon Rectum 1995;38:964-8

8. Classen M. Progress report - Fibroendoscopy of the intestine. Gut 1971;12:330-8

9. Dajani AS, Bisno AL, Chung KJ. Prevention of bacterial endocarditis: recommendations by American Heart Association. JAMA 1990;264:2919-22.

10. Ettersperger L, Zeitoun P, Thiefin G. Colonic perforations complicating colonoscopy. Apropos of 15 consecutive cases observed over 16 years. Gastroenterol Clin Biol 1995;19:1018-22.

11. Farley DR, Bannon MP, Zieltlow SP. Management of colonoscopic perforations. Mayo Clin Proc 1997;72:729-33.
12. Gorski TF, Rosen L, Riether R, Stasik J, Khubchandani I. Colorectal cancer after surveillance colonoscopy: false-negative examination or fast growth? Dis Colon Rectum 1999; 42:877-80.

13. Habr-Gama A. Emprego de solução de manitol a $10 \%$ no preparo do intestino grosso para colonoscopia e cirurgia. Rev Hosp Clin Fac Med S Paulo 1981;36:239-43.

14. Kozarek RA, Botoman VA, Patterson DJ. Prospective evaluation of a small caliber endoscope for colonoscopy after an unsuccessful standard examination. Gastrointest Endosc 1989;35:333-5.

15. Mckee CC, Ragland JJ, Myers JO. An evaluation of multiple clinical variables for hypoxia during colonoscopy. Surg Gynecol Obstet 1991;173:37-40.

16. Nagasko K, Takemoto T. Endoscopy of the ileo-cecal area. Gastroenterology 1973;65:403-11.

17. Nahas SC, Alves PA, Nahas PS, Habr-Gama A, Pinotti HW. Colonoscopia como método diagnóstico e terapêutico na doença do intestino grosso no Hospital das Clínicas da Faculdade de Medicina da Universidade de São Paulo [resumo]. Rev Bras Coloproctol 1989;9 (suppl. 1):20.

18. Nahas SC, Alves PRA, Araújo SEA. Emprego da colonoscopia como método diagnóstico e terapêutico das doenças do intestino grosso. Resultados observados em 1.715 exames. Rev Hosp Clin Fac Med S Paulo 1998;53:117-21.

19. Nahas SC, Lourenção JL, Borba M. Colonoscopia como método diagnóstico e terapêutico nas doenças do intestino grosso [resumo]. Rev Bras Coloproctol 1992;12 (suppl 1):41.

20. Nahas SC. Estudo comparativo entre os resultados obtidos com o emprego do enema opaco e da colonoscopia no diagnóstico das doenças do intestino grosso [resumo] Rev Bras Coloproctol 1989;9 (supl 1):43.

21. Nahas SC. Colonoscopia: método diagnóstico e terapêutico. Rev Hosp Clin Fac Med São Paulo 1995;50:154-9. 
22. Overholt BF, Pollard HM. Cancer of the colon and rectum. Current procedures for detection and diagnosis. Cancer 1967;20:445-50.

23. Parra-Blanco A, Kaminaga N, Kojima T, Endo Y, Tajiri A, Fujita R. Colonoscopy polypectomy with cutting current: is it safe? Gastrointest Endosc 2000;51:676-81.

24. Pérez Roldán F, González Carro P, Legaz Huidobro ML. Endoscopic resection of large colorectal polyps. Rev Esp Enferm Dig 2004;96:36-47.

25. Puchner R, Allinger S, Doblhofer F, Wallner M, Knoflach P. Complications of diagnostic and interventional colonoscopy. Wien Klin Wochenschr 1996;108:142-6.

26. Royal College of Physicians. Guidelines for training appraisal and assessment of trainees in GI endoscopy. London: RCP; 2001.

27. Sieg A, Hachmoeller-Eisenbach U, Eisenbach T. Prospective evaluation of complications in outpatient GI endoscopy: a survey among German gastroenterologists. Gastrointest Endosc 2001;53:620-7.

28. Standards of Training and Practice Commitee, American Society for Gastrointestinal Endoscopy. Infection control during gastrointestinal endoscopy: guidelines for clinical application. Gastrointest Endosc 1988;34 (supl. 37s).
29. Thomas-Gibson S, Thapar C, Shah SG, Saunders B. Colonoscopy at a combined district general hospital and specialist endoscopy unit: lessons from 505 consecutive examinations. J R Soc Med 2002;95:194-7.

30. Waye JD, Bashkoff E. Total colonoscoopy: is it always possible? Gastrointest Endosc 1991;37:152-4.

31. Waye JD, Kahin O, Auerbach ME. Complications of colonoscopy and flexible sigmoidoscopy. Gastrointest Clin North Am 1996;6:343-77.
Recebido em 8/3/2004 Aprovado em 30/11/2004 